\title{
Cost effectiveness of radiofrequency microremodeling for stress urinary incontinence
}

\author{
Peter K. Sand • Gary M. Owens • Edward J. Black • \\ Louise H. Anderson • Melissa S. Martinson
}

Received: 1 May 2013 / Accepted: 1 September 2013 / Published online: 10 October 2013

(C) The Author(s) 2013. This article is published with open access at Springerlink.com

\begin{abstract}
Introduction and hypothesis Stress urinary incontinence (SUI) is a common and growing problem among adult women and affects individuals and society through decreased quality of life (QoL), decreased work productivity, and increased health care costs. A new, nonsurgical treatment option has become available for women who have failed conservative therapy, but its cost effectiveness has not been evaluated. This study examined the cost effectiveness of transurethral radiofrequency microremodeling of the female bladder neck and proximal urethra compared with synthetic transobturator tape (TOT), retropubic transvaginal tape (TVT) sling, and Burch colposuspension surgeries for treating SUI.

Methods A Markov model was used to compare the cost effectiveness of five strategies for treating SUI for patients who had previously failed conservative therapy. The strategies were designed to compare the value of starting with a less invasive treatment. The cost-effectiveness analysis was conducted from the health care system perspective. Efficacy and adverse event rates were obtained from the literature; reimbursement costs were based on Medicare fee schedule. The model cycle was 3 months, with a 3-year time horizon. Single-variable sensitivity analyses were conducted to assess stability of base-case results.
\end{abstract}

\author{
P. K. Sand \\ University of Chicago, Chicago, USA \\ G. M. Owens \\ Gary Owens Associates, Glen Mills, PA, USA \\ E. J. Black $(\bowtie)$ \\ Reimbursement Strategies, LLC, 10287 Lancaster Bay, Saint Paul, \\ MN 55129-8527, USA \\ e-mail: black073@umn.edu
}

L. H. Anderson · M. S. Martinson

Technomics Research, LLC, Minneapolis, MN, USA
Results Two of the five strategies employed the use of transurethral radiofrequency microremodeling and achieved $17-$ $30 \%$ lower mean costs relative to their comparative sling or Burch strategies.

Conclusions Superior safety and cost effectiveness are recognized when patients are offered a sequential approach to SUI management that employs transurethral radiofrequency microremodeling before invasive surgical procedures. This sequential approach is consistent with treatment strategies for other conditions and offers a solution for women with SUI who want to avoid the inherent risks and costs of invasive continence surgery.

Keywords Burch · Cost effectiveness · Radiofrequency · Sling $\cdot$ Stress urinary incontinence $\cdot$ Transurethral

\section{Introduction}

Stress urinary incontinence (SUI), the most common form of UI, is defined by involuntary leakage of urine with effort, exertion, sneezing, or coughing [1]. Traditional surgical treatments include Burch colposuspension and autologous bladder-neck slings using rectus fascia, both of which have been used for many years. Subsequently, synthetic transobturator tape (TOT) and retropubic transvaginal tape (TVT) sling materials were introduced in kits, continuing to facilitate changing surgical approaches to SUI.

Recently, the US Food and Drug Administration (FDA) cleared a nonsurgical alternative developed for treating SUI. The Lyrette System ${ }^{\mathrm{TM}}$ was developed by Novasys Medical, Newark, CA, USA, and acquired by Verathon, Inc., Bothell, WA, USA. The procedure is referred to in this article as RFSUI: radiofrequency for stress urinary incontinence. This treatment uses a disposable RF probe that a physician passes through the urethra. RF energy is then used to generate 
controlled heat at low temperatures in tissue targets within the bladder neck and urethra. The heat denatures collagen in the tissue at the multiple treatment sites. Upon healing, the treated tissue demonstrates an increased resistance to involuntary leakage at times of heightened intra-abdominal pressure, thereby reducing or eliminating SUI episodes. RF-SUI can be performed in a physician's office or other outpatient setting.

Safety and efficacy of the RF-SUI procedure was demonstrated in three multicenter clinical trials [2-5]. Those trials indicated that RF collagen denaturation was not associated with any major adverse events (AE) and provided durable efficacy. In a blinded trial, 110 women were randomized to RF-SUI treatment and 63 to a sham treatment. After 12-month and 3-year follow-up, women with moderate to severe SUI reported greater increase (improvement) in Incontinence Quality of Life score compared with women who received the sham treatment $[2,3]$. In a prospective single-arm trial, 136 women were treated with RF-SUI and followed for 3 years, more than $60 \%$ of whom reported improved QoL and reduced symptoms, which continued through the follow-up period [4].

The introduction of a nonsurgical alternative to the treatment armamentarium for SUI offers one more option for physicians and patients. Although surgical and nonsurgical treatments are effective, some patients require multiple procedures before reaching clinical success as defined by dry rates. In this paper, we define a strategy as an intentionally staged series of treatments for SUI. The strategies were constructed to compare the costs and effectiveness of starting with a less invasive treatment versus starting with a surgical treatment.

Cost-effectiveness analysis has been used to compare interventions and provide decision support to identify which intervention offers good value for the money compared with alternatives. It is especially valuable when intervention strategies consist of multiple paths and multiple treatments over several years. RF-SUI treatment has lower costs and is associated with fewer serious AE (SAE) than surgical treatments, but clinical trials have shown that it is also associated with lower efficacy. Therefore, it is not clear whether the savings associated with RF-SUI in the short term will be maintained after several years, when subsequent SUI recurrence may require additional treatment. Cost-effectiveness models can incorporate the results of numerous randomized controlled trials (RCTs) to develop a broader picture of alternative strategies and their potential costs and effects. In this study, we developed a cost-effectiveness model to compare RF-SUI strategies to alternative surgical strategies for women with SUI who had failed conservative treatment. We report findings from the literature review for model inputs, the base case analysis, and sensitivity analysis.

\section{Materials and methods}

This study was an economic analysis with variable estimates from the literature. The study did not involve humans. Internal review board approval was not required. A Markov microsimulation model was developed to compare costs and effectiveness of five strategies for treating SUI. A Markov model is defined by a number of discrete health states, transition paths between states, transition cycle time, and total time horizon. A microsimulation projects a hypothetical patient along the transition paths to defined health states for the duration of the projection. In this study, a patient begins the simulation with a treatment for SUI and can then experience $\mathrm{AE}$, treatment success, treatment failure, and subsequent treatments. Costs and effects for each patient are assigned during each cycle and accumulated over the duration of the projection and over all simulated patients. The model evaluated RF-SUI strategies to surgical strategies for women with SUI who had failed conservative therapy. Two RF-SUI strategies, two sling strategies, and one Burch strategy was defined (Table 1). RF-SUI A was compared with Sling strategy A and Sling strategy B; RF-SUI strategy B was compared with Burch.

The strategies were developed based on the prevailing philosophy of the medical community and the American Urological Association (AUA) that care providers offer efficacious therapies and that first-line treatment should be conservative. If initial, conservative treatments fail, more invasive treatments may be considered. All simulated patients in this analysis were assumed to have failed conservative treatment. The RF-SUI procedure is minimally invasive and nonsurgical. We developed strategies to compare the costs and effects of

Table 1 Treatment strategies for stress urinary incontinence (SUI)

\begin{tabular}{|c|c|c|c|}
\hline Strategy & $\begin{array}{l}\text { Primary } \\
\text { treatment }\end{array}$ & $\begin{array}{l}\text { Secondary } \\
\text { treatment, } \\
\text { if needed }\end{array}$ & $\begin{array}{l}\text { Tertiary treatment, } \\
\text { if needed }\end{array}$ \\
\hline \multirow[t]{2}{*}{ RF-SUI strategy A } & RF-SUI & TVT $(40 \%)$ & TVT (50\% \\
\hline & & TOT (60\%) & Traditional (50\%) \\
\hline \multirow[t]{2}{*}{ Sling strategy A } & TVT $(40 \%)$ & $\begin{array}{l}\text { TVT }(50 \% \\
\text { Traditional } \\
\quad(50 \%)\end{array}$ & Traditional \\
\hline & TOT $(60 \%)$ & TVT & Traditional \\
\hline Sling strategy B & $\begin{array}{l}\text { TVT }(40 \%) \\
\text { TOT }(60 \%)\end{array}$ & Burch & Burch \\
\hline RF-SUI strategy B & RF-SUI & Burch & $\begin{array}{l}\text { TVT }(40 \%) \\
\text { TOT }(60 \%)\end{array}$ \\
\hline Burch strategy & Burch & $\begin{array}{l}\text { TVT }(40 \%) \\
\text { TOT (60 \%) }\end{array}$ & $\begin{array}{l}\text { TVT (50\% } \\
\text { Traditional (50\%) }\end{array}$ \\
\hline
\end{tabular}

$R F$-SUI radiofrequency for stress urinary incontinence, $T V T$ retropubic midurethral synthetic sling, TOT transobturator midurethral synthetic sling, Traditional bladder-neck autologous sling, Burch Burch colposuspension 
using the RF-SUI treatment with strategies starting with a surgical intervention. The subsequent treatment in each strategy was based on practice patterns in North America. For a patient who failed RF-SUI, most surgeons would proceed to a minimally invasive procedure, such as a midurethral sling. Because some surgeons or patients have concerns about complications associated with synthetic mesh, they may proceed directly to a Burch procedure. When midurethral slings fail, it is often due to intrinsic sphincter insufficiency, which would lead the surgeon to recommend retropubic midurethral slings. Should retropubic slings fail, it could be due to a lack of urethral hypermobility or significant sphincter insufficiency. Most surgeons would follow a failed retropubic sling with a traditional autologous bladder-neck sling.

\section{Model structure}

For each treatment strategy, outcomes for 100,000 simulated patients were estimated over a 3-year time horizon in quarterly cycles. Patients entering the model were assumed to have failed previous conservative treatment for SUI. At the beginning of each simulation, patients received the primary treatment as defined by the strategy. At each subsequent cycle, the patient could follow one of four paths: (1) experience an SAE, (2) experience a minor adverse event, (3) experience treatment failure, or (4) continue with ongoing monitoring of care. Transition probabilities were based on published evidence; the proportion of TVT and TOT treatments was assumed to be $40 \%$ and $60 \%$, respectively, based on experience of the first author. AE incurred treatment costs and returned to treatment follow-up in the next cycle. Treatment failures were followed by secondary or tertiary treatment, as shown in Table 1. This is a worst-case scenario of retreatment and therefore of costs. In actual clinical practice, not all patients who experience treatment failure as measured by pad-weight testing seek additional therapy. Effectiveness was measured by the number of quarters in which primary treatment maintained efficacy or the end of the simulation. Costs were evaluated from the perspective of the health care system and included reimbursement to hospitals and physicians for treating SUI, treating AE, and follow-up care. Indirect costs for patient time were not considered. Costs and outcomes were recorded at each cycle of the simulation and discounted at an annual rate of $5 \%$. The model was developed using TreeAge Pro 2012 (TreeAge Software, Inc., Williamstown, MA, USA).

\section{Model inputs}

Model inputs were identified from the literature. Key assumptions in the model were treatment efficacy, AE associated with treatment, and health care costs of treatment and AE. The purpose of the analysis was to compare costs and effects of the defined treatment strategies; therefore, efficacy, AE, and cost assumptions were carefully evaluated. Literature sources for estimates of treatment efficacy and incidence of AE were identified through a MEDLINE search and references from Cochrane Reviews of surgical treatment for SUI [6, 7]. Pad test was selected as the efficacy measure because it was the most consistently reported measure across studies. Estimates of AE were taken from recent multicenter RCTs of SUI treatment, which provided complete AE reporting [2, 4, 8-13]. AE that resulted in additional health care costs were included in the incidence rate. AE that occurred intraoperatively, were unrelated to treatment, or were not defined specifically enough to identify treatment were excluded. Loss of efficacy and AE incidence from each study were converted to quarterly rates assuming a standard exponential distribution. Rates were averaged across studies weighted by number of patients. Studies were excluded if they contained patients with intrinsic sphincter deficiency or urgency urinary incontinence (UUI), reported $<12$ month of follow-up, were not written in the English language, or were reported in an Abstract only. Studies that did not report efficacy based on pad test were also excluded.

All payments were based on the Medicare 2012 fee schedule allowance. RF-SUI procedures were assumed to be performed as an office visit; sling procedures were assumed to be performed as outpatient - one third in ambulatory surgery centers and two thirds in hospital outpatient facilities; and Burch surgeries were assumed to be performed as hospital inpatient only. Ambulatory surgical centers are free-standing facilities that provide same-day surgical care and are an alternative to hospital outpatient facilities. Follow-up office visits were assumed to occur 6-months and 12-months postprocedure and annually thereafter. Average costs for treating serious and minor AE were estimated for each SUI treatment. Types and numbers of events were identified from the literature [2, 4, 8-13]. The average cost for treating serious and minor AE was weighted by the number of events reported.

\section{Sensitivity analysis}

Single-variable sensitivity analyses were used to assess stability of base-case results. The simulation was repeated using a range of values for key variables, and results were compared with those from the base case. The lower and upper limits of a normal $95 \%$ confidence interval (CI) were used to evaluate sensitivity to efficacy and AE rates. Costs for procedures and treating AE were assumed to range $\pm 10 \%$ of base case for RFSUI and from $+20 \%$ to $-10 \%$ of base case for the other treatments. The difference in range was due to the complexity of the treatments. RF-SUI is a relatively simple treatment with no facility or independent anesthesiology expenses. The complexity of the other treatments warranted higher variable costs because of the additional input costs from ambulatory surgery 
centers, hospitals, postoperative pain management, and regional variations in physician utilization of ancillary resources.

\section{Results}

\section{Literature review}

Eight articles representing seven studies were included for estimates of treatment efficacy (Table 2) [4, 9, 11, 12, 14-17]. Efficacy measured by pad test ranged from $35 \%$ over a 36-month period [4] to $92 \%$ over a 12 -month period [12]. Four studies used a 1-h pad test and defined dry as $<1$ or $<2 \mathrm{~g}$, one used a 20-min pad test, and two used a 24-h pad test. Three studies had follow-up periods of 12 months; the others measured outcomes at 24,36 , or 60 months.

Serious and minor AE rates were estimated from five studies [2, 4, 8-13]. The most common SAEs resulting in additional costs were voiding dysfunction leading to surgical revision, wound complication requiring surgical intervention,

Table 2 Efficacy estimates from reviewed literature

\begin{tabular}{|c|c|c|c|}
\hline & \multicolumn{3}{|l|}{ Pad test } \\
\hline & Efficacy & Patients & Follow-up period \\
\hline \multicolumn{4}{|c|}{ Transurethral radiofrequency energy collagen microremodeling } \\
\hline Elser $2011[4]$ & $35 \%{ }^{\mathrm{a}}$ & 136 & 36 months \\
\hline \multicolumn{4}{|c|}{ TVT, retropubic midurethral synthetic sling } \\
\hline Liapis 2002 [14] & $84 \%{ }^{\mathrm{b}}$ & 36 & 24 months \\
\hline Liapis 2006 [15] & $89 \%{ }^{\mathrm{b}}$ & 46 & 12 months \\
\hline Richter 2010 [12] & $92.3 \%^{\mathrm{c}}$ & 298 & 12 months \\
\hline Wang 2003 [16] & $81.6 \%{ }^{\mathrm{d}}$ & 49 & 12 months \\
\hline Ward 2004 [11] & $77.7 \%{ }^{\mathrm{b}}$ & 175 & 24 months \\
\hline Ward $2007[17]$ & $74.9 \%{ }^{\mathrm{b}}$ & 175 & 5 years \\
\hline \multicolumn{4}{|c|}{ TOT, transobturator midurethral synthetic sling } \\
\hline Liapis $2006[15]$ & $90 \%{ }^{\mathrm{b}}$ & 43 & 12 months \\
\hline Richter 2010 [12] & $93 \%{ }^{\mathrm{c}}$ & 299 & 12 months \\
\hline \multicolumn{4}{|c|}{ Burch colposuspension surgery } \\
\hline Albo 2007 [9] & $85 \%{ }^{\mathrm{c}}$ & 255 & 24 months \\
\hline Liapis 2002 [14] & $86^{\mathrm{b}}{ }^{\mathrm{b}}$ & 35 & 24 months \\
\hline Ward $2004[11]$ & $68 \%{ }^{\mathrm{b}}$ & 169 & 24 months \\
\hline Ward 2007 [17] & $69 \%{ }^{\mathrm{b}}$ & 169 & 5 years \\
\hline \multicolumn{4}{|c|}{ Traditional bladder-neck autologous sling } \\
\hline Albo 2007 [9] & $86 \%^{\mathrm{c}}$ & 265 & 24 months \\
\hline
\end{tabular}

Two- and 5-year results from Ward 2004 and Ward 2007 were interpolated to estimate a 3-year measure for modeling

a 20 -min pad test, dry pad measured as $<1 \mathrm{~g}$

${ }^{\mathrm{b}} 1$-h pad test, dry pad measured as $<1 \mathrm{~g}$

${ }^{\mathrm{c}} 24$-h pad test, dry pad measured as $<15 \mathrm{~g}$

${ }^{\mathrm{d}} 1$-h pad test, dry pad measured as $<2 \mathrm{~g}$ mesh exposure or erosion, and recurrent cystitis leading to diagnostic cystoscopy. Cystitis and wound complication not requiring surgical intervention were common minor AE.

\section{Base case}

Base-case assumptions for each SUI treatment are shown in Table 3. Annual loss-of-efficacy rates for sling treatments and Burch surgery were similar at $7-10 \%$. The rate for RF-SUI was $35 \%$; however, the annual minor AE rate was lower than all other treatments, and the annual SAE rate was zero. Burch surgery and traditional slings had the highest rates of minor AE; TVT, traditional slings, and Burch surgery had the highest rates of SAEs at $3-6 \%$.

Procedure costs for RF-SUI were less than one half the cost of sling treatments and were one fifth the cost of Burch surgery. Average treatment costs for SAEs were similar for sling and Burch surgery. Average treatment costs for minor AE were lowest for RF-SUI and traditional slings and highest for TVT and TOT slings. The cost of follow-up office visits was assumed to be the same for all SUI treatments.

Results of the base-case simulation for the five strategies are shown in Table 4. Compared with the sling strategies, RF-SUI strategy A had the lowest average costs over the 3-year period (\$4,102), $17 \%$ lower than Sling strategy A and $29 \%$ lower than Sling strategy B. Compared with the Burch strategy, RF-SUI strategy B had $30 \%$ lower average costs over the 3 -year period $(\$ 6,579)$. The average duration of primary treatment efficacy was 8 quarters for both of the RF-SUI strategies and 11 quarters for the Sling and Burch strategies, with the maximum of 12 quarters. At the end of the 3-year simulation, about $80 \%$ of sling and Burch strategy patients had not received secondary treatment: $36 \%$ of RF-SUI strategy patients had not received secondary treatment. Less than $3 \%$ of patients in the RF-SUI strategy A or B groups experienced an SAE; more than three times that number experienced SAEs in the other strategies.

Sensitivity analysis

In the sensitivity analysis, efficacy and procedure costs were the most influential on total costs. AE rates and cost had little impact on results. When all treatments were at their upper or lower limit of efficacy, the relative ranking of strategies by mean cost did not change from the base case. RF-SUI strategy A (\$2,970 upper limit; $\$ 4,973$ lower limit) was lower than sling A $(\$ 4,477 ; \$ 5,427)$ and B $(\$ 4,765 ; \$ 6,809)$ strategies. RF-SUI strategy B $(\$ 4,347 ; \$ 8,146)$ was lower than the Burch strategy $(\$ 8,989 ; \$ 9,897)$. When RF-SUI was at the upper limit of efficacy and the other treatments were at their lower limits, RF-SUI strategies were $43-55 \%$ less expensive than the comparative sling and Burch strategies. When RF-SUI was at its lower limit of efficacy and the other treatments were at their upper limits, costs of RF-SUI strategies remained 
Table 3 Base-case assumptions

\begin{tabular}{|c|c|c|c|c|c|c|}
\hline \multirow[b]{2}{*}{ Treatment } & \multicolumn{3}{|l|}{ Annual rates } & \multicolumn{3}{|c|}{ Reimbursement costs } \\
\hline & Loss of efficacy & Minor AE & Serious AE & Procedure & Minor AE & Serious AE \\
\hline RF-SUI & 0.350 & 0.135 & 0.000 & $\$ 1,571.74$ & $\$ 57.55$ & \\
\hline TVT synthetic & 0.096 & 0.391 & 0.052 & $\$ 3,735.84$ & $\$ 154.79$ & $\$ 3,754.13$ \\
\hline TOT synthetic & 0.077 & 0.159 & 0.017 & $\$ 3,735.84$ & $\$ 138.27$ & $\$ 2,999.04$ \\
\hline Burch surgery & 0.097 & 0.899 & 0.037 & $\$ 8,061.10$ & $\$ 68.56$ & $\$ 3,235.48$ \\
\hline Traditional sling & 0.075 & 0.890 & 0.062 & $\$ 3,735.84$ & $\$ 57.55$ & $\$ 2,981.99$ \\
\hline
\end{tabular}

AE adverse events, RF-SUI radiofrequency for stress urinary incontinence, TVT retropubic midurethral synthetic sling, TOT transobturator midurethral synthetic sling, Traditional bladder-neck autologous sling

Follow-up office visit reimbursement $\$ 42.44$ for all treatments

lower compared with Sling strategy B and Burch, but RF-SUI strategy A was $4 \%$ higher than Sling strategy A. Comparing across strategies at the upper limits of procedure cost maintained the relationship found in the base-case analysis. RF-SUI strategies were $19-31 \%$ lower in mean cost relative to their comparative sling or Burch strategies.

\section{Discussion}

This study compares the costs and effectiveness of five strategies used to treat female SUI. Comparisons are between the less invasive, office-based RF-SUI and commonly used surgical interventions such a midurethral slings (TVT or TOT) and retropubic colposuspensions (Burch). Although the AUA and the International Urogynecological Association (IUGA) [18] have proposed standards for reporting efficacy and outcomes in SUI trials, these standards have not been universally adopted and used in many trials frequently referenced in the SUI literature. Given the variety of outcome definitions used to define success across a multitude of SUI studies, we selected pad weight as the objective outcome, as this is thought to be the most consistent measurement used across the studies referenced in this paper.

It should be understood that measuring efficacy only in terms of outcomes does not represent the fully burdened cost of treatment because it does not incorporate costs of potential complications. The addition of costs associated with managing SAEs in each treatment arm was therefore included in this analysis. The materiality of such costs impacting the shortand long-term management of such SAEs serves to offset some of the qualitative and quantitative success rates associated with the use of the different treatment modalities. The addition of such AE costs are thought to be a valuable contribution to payers in light of the differences noted in the SAE profiles among the treatment strategies discussed.

Table 4 Simulation results; comparison of treatment strategies

\begin{tabular}{|c|c|c|c|c|}
\hline Strategies & Mean cost & $\begin{array}{l}\text { Average duration of primary } \\
\text { treatment, quarters }{ }^{\mathrm{a}}, \max =12\end{array}$ & $\begin{array}{l}\text { Percent still on primary } \\
\text { procedure at } 3 \text { years }\end{array}$ & $\begin{array}{l}\text { Percent with } \\
\text { SAEs at } 3 \text { years }\end{array}$ \\
\hline RF-SUI strategy A & $\$ 4,102$ & 8 & $36 \%$ & $2.4 \%$ \\
\hline Sling strategy A & $\$ 4,962$ & 11 & $79 \%$ & $8.3 \%$ \\
\hline Sling strategy B & $\$ 5,807$ & 11 & $79 \%$ & $8.0 \%$ \\
\hline RF-SUI strategy B & $\$ 6,579$ & 8 & $36 \%$ & $2.9 \%$ \\
\hline \multirow[t]{2}{*}{ Burch strategy } & $\$ 9,450$ & 11 & $76 \%$ & $9.3 \%$ \\
\hline & Initial & Secondary & Tertiary & \\
\hline RF-SUI strategy A & RF-SUI $\rightarrow$ & TVT or TOT $\rightarrow$ & TVT or traditional & \\
\hline Sling strategy A & TVT or TOT $\rightarrow$ & TOT or traditional or TVT $\rightarrow$ & Traditional & \\
\hline Sling strategy B & TVT or TOT $\rightarrow$ & Burch $\rightarrow$ & Burch & \\
\hline RF-SUI strategy B & RF-SUI $\rightarrow$ & Burch $\rightarrow$ & TOT or TOT & \\
\hline Burch strategy & Burch $\rightarrow$ & TVT or TOT $\rightarrow$ & TVT or traditional & \\
\hline
\end{tabular}

$R F-S U I$ radiofrequency for stress urinary incontinence, TVT retropubic midurethral synthetic sling, TOT transobturator midurethral synthetic sling, Traditional bladder-neck autologous sling, SAE serious adverse event

${ }^{a}$ Average duration over the 12-quarter simulation is the average of the number of quarters that each simulated patient went before there was a failure of efficacy based on pad weight 
In comparing the costs of the different treatment strategies, a gradual but persistent increase in payer cost is noted over a 3 -year period as the intensity of the therapies increases. RFSUI strategy A has the lowest cost, at $\$ 4,100$, followed by an increase in cost with sling surgeries ranging from $\$ 5,000$ to $\$ 5,800$ and culminating in the highest cost, the Burch procedure, at $\$ 9,500$. And although it is clear that complexity, costs, and success rates are highly correlated, it should also be noted that the SAE rates and costs associated with managing these SAEs are also highly correlated. Therefore, just as the efficacy costs of the five treatment strategies are identified in this paper, so, too, are SAE costs of these five treatment strategies. Again, it is clear that as complexity and costs increase, so do costs of managing both transient and life-altering AE.

As a general prevailing philosophy, most physicians and providers offer patients therapies with proven efficacy and try nonsurgical treatments first. If those treatments are not successful, patients and their physicians may consider more invasive, hence more risky, procedures. For example, that philosophy has been outlined by the AUA for treating overactive bladder [19, 20]. It is that philosophy we used to develop strategies for treating SUI in women who failed conservative therapy. We compared strategies that incorporate the use of nonsurgical RFSUI before moving to surgery. Inclusion of RF-SUI was expected to improve outcomes for some women without subjecting them to surgery and to reduce overall health care costs. The suggestion that cost savings can be identified if physicians and patients are encouraged to follow a sequential strategy for managing SUI first by using the least-invasive, lower-cost, safer procedure, is supported by the findings of this study. A recent health technology assessment by Imamura et al. was consistent with findings that care paths that begin with conservative, office-based treatments are more cost effective than initial operative treatment or a rapid progression to operative therapy [21]. The application of such progressively invasive treatment strategies to SUI management results in a direct per-patient treatment savings of $\$ 1,000$ over a 3-year period if patients are treated with the least invasive therapy first.

Whereas treatment with RF-SUI may be successful for some women, others will not have satisfactory results and will opt for a subsequent surgical procedure. The previous use of RF-SUI will not interfere with subsequent surgery and in fact may improve surgical results. The lesions created during RFSUI treatment are microlesions within the urethra. Surgical procedures are performed outside the urethra, and the microlesions will not inhibit surgical success. In addition, Appell et al. found that RF-SUI improved leak-point pressure, which would serve to enhance the outcome of surgery [2].

Whereas some women may simply prefer noninvasive treatments, others may be concerned over recent alerts related to synthetic mesh. On 20 October 2008, the FDA issued a Public Health Notification on serious complications associated with surgical mesh placed through the vagina (transvaginal placement) to treat POP [22]. To evaluate the safety and effectiveness of surgical mesh, the FDA conducted a review of the scientific literature from 1996 through 2011. The review concluded that the use of mesh for POP repair did not improve symptoms or QoL over traditional nonmesh repair. The review of surgical mesh use in SUI treatment is ongoing, with an FDA report to be issued at a later date. The AUA believes the true incidence rate for SAEs associated with synthetic mesh is not known [23].

The major limitations in this analysis are similar to those in other SUI studies: absence of consistent terminology and definitions used to define qualitative and quantitative outcomes, lack of a uniform and standardized definition of success, lack of RCTs with and without blinding of the five different treatment arms used in this analysis, variability in time horizons for patient follow-up, and high lost-to-follow-up rate in all studies for patients followed $>1$ year. The addition of qualitative or subjective outcome measures was initially considered for use in this study; however, it was discovered that these measures of success and patient-reported outcomes also varied widely across trials. Randomized studies would ensure that patients undergoing surgical procedures or RF-SUI were equivalent and that efficacy results used in this analysis were applicable to the same types of patients. However, real-world outcomes may vary from results observed in RCTs, which are often performed in academic medical centers by seasoned, experienced surgeons. For modeling purposes, we assumed that patient characteristics in the study were similar, as they were all refractory to conservative therapy prior to more significant interventions, such as RF-SUI, slings, or Burch procedures.

In our literature review, we selected studies that included patients who would be candidates for RF-SUI and surgical treatments assessed in the model. Because RF-SUI is not recommended for patients with intrinsic sphincter deficiency or urgency urinary incontinence, studies for those patients were excluded. Our decision to base treatment efficacy on the pad test was driven by the frequency and consistency with which that test was reported in the literature. Use of the pad test as an accepted measure of success is supported by its use as an outcome by three major multicenter RCTs for surgical treatments [9-12]. Although consistency of objective outcome measures is lacking, measures tend to result in similar conclusions: a procedure that is efficacious when measured with the pad test is also efficacious when measured with leak-point pressure. Because of that tendency, we believe our choice of pad test for efficacy should not have biased results. However, subjective measures of success, such as increase in QoL or self-report diaries, may be influenced by patient expectations and experience. Attempts to extend this analysis beyond this 3 -year time horizon was limited by the absence of long-term studies, high levels of patient fallout in the studies evaluated, and the absence of meaningful cross-functional, quantitative parameters by which studies could be compared. We believe 
the analysis and the 3-year time horizon used is sufficiently robust to provide valid conclusions.

\section{Conclusion}

SUI has a profound negative effect on QoL of affected individuals and a negative impact on society with increased health care costs and decreased work productivity. SUI management strategies presented here are supported by the literature and offer physicians and health policymakers a solution for women with SUI who want to avoid the inherent risks and recovery of invasive continence surgery. Superior safety and cost effectiveness are recognized when patients are offered a sequential approach to SUI management. This sequential approach is consistent with treatment strategies for other conditions in a health care environment that pursues both quality and cost effectiveness as valuable attributes in the provision of patient care.

Funding Source This study was funded in part by Novasys Medical of Newark, CA, USA. The study sponsor had no role in the study design, data collection, data analysis, interpretation of results, or writing of this manuscript.

Conflicts of Interest P.K. Sand: Consultancies or paid advisory board to Allergan, Astellas, Ferring, Merck, Pfizer, Uroplasty, Watson; grants received from Boston Scientific, Allergan, Ferring; Honoraria from Allergan, Astellas; Lecture fees from Allergan, Astellas, Pfizer, Watson.

G.M. Owens: Consultant to Novasys Medical.

E.J. Black: Consultant to Novasys Medical, paid consultant on this project.

L.H. Anderson: Consultant to Novasys Medical, paid consultant on this project.

M.S. Martinson: Consultant to Novasys Medical, paid consultant on this project.

Open Access This article is distributed under the terms of the Creative Commons Attribution License which permits any use, distribution, and reproduction in any medium, provided the original author(s) and the source are credited.

\section{References}

1. Haylen BT, de Ridder D, Freeman RM et al (2010) An International Urogynecological Association (IUGA)/International Continence Society (ICS) joint report on the terminology for female pelvic floor dysfunction. Int Urogynecol J 21:5-26. doi:10.1007/s00192-009-0976-9

2. Appell RA, Juma S, Wells WG et al (2006) Transurethral radiofrequency energy collagen micro-remodeling for the treatment of female stress urinary incontinence. Neurourol Urodyn 25:331336. doi:10.1002/nau.20185

3. Appell RA, Singh G, Klimberg IW et al (2007) Nonsurgical, radiofrequency collagen denaturation for stress urinary incontinence: retrospective 3-year evaluation. Expert Rev Med Devices 4:455-461. doi:10.1586/17434440.4.4.455

4. Elser DM, Mitchell GK, Miklos JR et al (2011) Nonsurgical transurethral radiofrequency collagen denaturation: results at three years after treatment. Adv Urol 2011:872057. doi:10.1155/2011/872057
5. Sotomayor M, Bernal GF (2005) Twelve-month results of nonsurgical radiofrequency energy micro-remodeling for stress incontinence. Int Urogynecol J Pelvic Floor Dysfunct 16:192-196. doi: 10.1007/s00192-004-1223-Z

6. Ogah J, Cody JD, Rogerson L (2009) Minimally invasive synthetic suburethral sling operations for stress urinary incontinence in women. Cochrane Database Syst Rev. doi:10.1002/14651858.CD006375.pub2

7. Rehman H, Bezerra CC, Bruschini H, Cody JD (2011) Traditional suburethral sling operations for urinary incontinence in women. Cochrane Database Syst Rev. doi:10.1002/14651858.CD001754.pub3

8. Elser DM, Mitchell GK, Miklos JR et al (2009) Nonsurgical transurethral collagen denaturation for stress urinary incontinence in women: 12-month results from a prospective long-term study. J Minim Invasive Gynecol 16:56-62. doi:10.1016/j.jmig.2008.09.621

9. Albo ME, Richter HE, Brubaker L et al (2007) Burch colposuspension versus fascial sling to reduce urinary stress incontinence. N Engl J Med 356:2143-2155. doi:10.1056/NEJMoa070416

10. Ward K, Hilton P (2002) Prospective multicentre randomised trial of tension-free vaginal tape and colposuspension as primary treatment for stress incontinence. BMJ 325:67

11. Ward KL, Hilton $P$ (2004) A prospective multicenter randomized trial of tension-free vaginal tape and colposuspension for primary urodynamic stress incontinence: two-year follow-up. Am J Obstet Gynecol 190:324-331. doi:10.1016/j.ajog.2003.07.029

12. Richter HE, Albo ME, Zyczynski HM et al (2010) Retropubic versus transobturator midurethral slings for stress incontinence. N Engl J Med 362:2066-2076. doi:10.1056/NEJMoa0912658

13. Brubaker L, Norton PA, Albo ME et al (2011) Adverse events over two years after retropubic or transobturator midurethral sling surgery: findings from the Trial of Midurethral Slings (TOMUS) study. Am J Obstet Gynecol 205:498.e1-6. doi:10.1016/j.ajog.2011.07.011

14. Liapis A, Bakas P, Creatsas G (2002) Burch colposuspension and tension-free vaginal tape in the management of stress urinary incontinence in women. Eur Urol 41:469-473

15. Liapis A, Bakas P, Giner M, Creatsas G (2006) Tension-free vaginal tape versus tension-free vaginal tape obturator in women with stress urinary incontinence. Gynecol Obstet Invest 62:160-164. doi:10. $1159 / 000093320$

16. Wang AC, Chen M-C (2003) Comparison of tension-free vaginal taping versus modified Burch colposuspension on urethral obstruction: a randomized controlled trial. Neurourol Urodyn 22:185-190. doi:10.1002/nau.10092

17. Ward KL, Hilton P (2008) Tension-free vaginal tape versus colposuspension for primary urodynamic stress incontinence: 5-year follow up. BJOG 115:226-233. doi:10.1111/j.1471-0528.2007.01548.x

18. Ghoniem G, Stanford E, Kenton K et al (2008) Evaluation and outcome measures in the treatment of female urinary stress incontinence: International Urogynecological Association (IUGA) guidelines for research and clinical practice. Int Urogynecol J Pelvic Floor Dysfunct 19:5-33. doi:10.1007/s00192-007-0495-5

19. Gormley EA, Lightner DJ, Burgio KL et al (2012) Diagnosis and treatment of overactive bladder (non-neurogenic) in adults: AUA/ SUFU guideline. J Urol 188:2455-2463. doi:10.1016/j.juro.2012.09.079

20. Brown T (2012) Overactive bladder guidelines released. Medscape Medical News

21. Imamura M, Abrams $P, B a i n ~ C$ et al (2010) Systematic review and economic modelling of the effectiveness and cost-effectiveness of non-surgical treatments for women with stress urinary incontinence. Health Technol Assess 14:1-188. doi:10.3310/hta14400, iii-iv

22. FDA Alerts and Notices (Medical Devices) - FDA safety communication: UPDATE on serious complications associated with Transvaginal placement of surgical mesh for pelvic organ prolapse. http://www.fda.gov/medicaldevices/safety/alertsandnotices/ ucm262435.htm. Accessed 20 Jun 2012

23. AUA American Urological Association Clinical Practice Guidelines. Chapter 3, p 11 